\title{
Solar Mikheyev-Smirnov-Wolfenstein effect with three generations of neutrinos
}

\author{
Per Osland \\ Department of Physics, University of Bergen, Allégaten 55, N-5007 Bergen, Norway \\ Tai Tsun Wu \\ Gordon McKay Laboratory, Harvard University, Cambridge, Massachusetts 02138 \\ and Theoretical Physics Division, CERN, CH-1211 Geneva 23, Switzerland
}

(Received 29 December 1999; published 7 June 2000)

\begin{abstract}
Under the assumption that the density variation of the electrons can be approximated by an exponential function, the solar Mikheyev-Smirnov-Wolfenstein effect is treated for three generations of neutrinos. The generalized hypergeometric functions that result from the exact solution of this problem are studied in detail, and a method for their numerical evaluation is presented. This analysis plays a central role in the determination of neutrino masses, not only the differences of their squares, under the assumption of universal quark-lepton mixing.

PACS number(s): 14.60.Pq, 13.15.+g
\end{abstract}

\section{INTRODUCTION}

Recently a program has been proposed to study neutrino masses [1]. This program is based on the following assumptions.

Assumption 1: There are three right-handed Dirac neutrinos in addition to the three known left-handed neutrinos.

Assumption 2: The lepton mass matrices have the same structure as the quark mass matrices, as described by Lehmann et al. [2].

In carrying out this program, one of the technically most difficult aspects is to treat the Mikheyev-SmirnovWolfenstein (MSW) effect for the Sun [3]. It is the purpose of this paper to discuss this problem. ${ }^{1}$

The difficulty arises in the following way. On the basis of the Super-Kamiokande data on atmospheric neutrinos [4], the muon neutrino $\nu_{\mu}$ oscillates with another neutrino which is not the electron neutrino $\nu_{e}$. From assumption 1, this neutrino must be the tau neutrino $\nu_{\tau}$. In other words, $\nu_{\mu}$ and $\nu_{\tau}$ are coupled significantly. Again from assumption 1, the electron neutrinos $\nu_{e}$ from the Sun must therefore oscillate with either $\nu_{\mu}$ or $\nu_{\tau}$. Since $\nu_{\mu}$ and $\nu_{\tau}$ are coupled significantly, the electron neutrinos $\nu_{e}$ from the Sun must oscillate with both $\nu_{\mu}$ and $\nu_{\tau}$. It is accordingly necessary to understand the MSW effect with all three generations of neutrinos $\nu_{e}, \nu_{\mu}$ and $\nu_{\tau}$.

While there is an extensive literature on the MSW effect for two generations of neutrinos [5,6], relatively little is known for the case of three generations [7]. One of the major differences between these two cases is the following. For two generations, the MSW effect occurs predominantly in the vicinity of a particular density in the Sun. For this reason, the important parameter is the rate of change of the density at this particular density. In contrast, for three generations, the important contributions to the MSW effect can come from

\footnotetext{
${ }^{1}$ The solution of the evolution equation, presented in this paper, does not rely on assumption 2 .
}

several regions. When the parameters, such as the neutrino masses, vary, these regions not only move but also merge and separate, features that are absent for the much simpler case of two generations.

\section{FORMULATION OF THE PROBLEM}

There are many different ways to formulate the problem of the MSW [3] effect in the sun for three generations of neutrinos. These different formulations are of course closely related to each other; it has been found that, at least for the present program [1], the following one is most appropriate.

In a current basis, the charged lepton currents are

$$
J_{\mu} \sim \bar{\nu}_{\mathrm{L}} \gamma_{\mu} l_{\mathrm{L}}
$$

where

$$
\nu=\left[\begin{array}{c}
\nu_{1} \\
\nu_{2} \\
\nu_{3}
\end{array}\right] \quad \text { and } \quad l=\left[\begin{array}{c}
l_{1} \\
l_{2} \\
l_{3}
\end{array}\right]
$$

denote the neutrino and charged lepton fields. The neutrino mass matrix $M$ [8], from the vacuum expectation value of the Higgs field in the neutrino-Higgs coupling, is

$$
\bar{\nu}_{\mathrm{L}} M \nu_{\mathrm{R}}+\text { H.c. }
$$

Let $p$ be the momentum of the neutrino, the value of which is taken to be much larger than the neutrino masses, which are the eigenvalues of $M$. Typically, $p$ is in the range from $0.4 \mathrm{MeV} / c$ to $10 \mathrm{MeV} / c$, while the neutrino masses are believed to be no more than about $1 \mathrm{eV}$; hence this assumption is well satisfied. Under this assumption, it is $M^{2}$ that enters in the MSW effect. Let 


$$
M^{2}=\left[\begin{array}{ccc}
M_{11}^{2} & M_{12}^{2} & M_{13}^{2} \\
M_{21}^{2} & M_{22}^{2} & M_{23}^{2} \\
M_{31}^{2} & M_{32}^{2} & M_{33}^{2}
\end{array}\right]
$$

be real and symmetric. Note that $M_{i j}$ are not defined, only $M_{i j}^{2} \equiv\left(M^{2}\right)_{i j}$. Of course the eigenvalues of this matrix $M^{2}$ are the squares of the neutrino masses. ${ }^{2}$

In terms of this $M^{2}$, the MSW effect for neutrino oscillations is described by the coupled ordinary differential equation [3]

$$
\begin{aligned}
i \frac{d}{d r}\left[\begin{array}{l}
\phi_{1}(r) \\
\phi_{2}(r) \\
\phi_{3}(r)
\end{array}\right]= & \left(\left[\begin{array}{ccc}
D(r) & 0 & 0 \\
0 & 0 & 0 \\
0 & 0 & 0
\end{array}\right]\right. \\
& \left.+\frac{1}{2 p}\left[\begin{array}{lll}
M_{11}^{2} & M_{12}^{2} & M_{13}^{2} \\
M_{21}^{2} & M_{22}^{2} & M_{23}^{2} \\
M_{31}^{2} & M_{32}^{2} & M_{33}^{2}
\end{array}\right]\right)\left[\begin{array}{l}
\phi_{1}(r) \\
\phi_{2}(r) \\
\phi_{3}(r)
\end{array}\right],
\end{aligned}
$$

where

$$
D(r)=\sqrt{2} G_{\mathrm{F}} N_{e}(r),
$$

with $G_{\mathrm{F}}$ the Fermi weak-interaction constant and $N_{e}(r)$ the electron density at the point $r$.

So far this formulation is general. In view of the observation in Sec. I that, for three generations of neutrinos, important contributions to the MSW effect can come from several values of $r$, we propose to choose a suitable function $D(r)$. On the one hand, this $D(r)$ should describe approximately the electron density in the sun; on the other hand, this $D(r)$ should be sufficiently simple so that Eq. (2.4) can be solved explicitly. This choice is made in the following way.

First, since most neutrinos are produced by nuclear reactions [9] near the center of the Sun, the simplifying assumption is made that the neutrinos are produced at the center of the Sun. This has the consequence that the $r$ in Eqs. (2.4) and (2.5) is the radial distance from the center of the Sun. This is the reason for the notation $r$.

Secondly, the density of the sun as a function of the radial distance is fairly accurately known [10], as shown by the solid curve in Fig. 1, where the vertical axis is the density in $\mathrm{g} / \mathrm{cm}^{3}$ in a logarithmic scale, and the horizontal axis is $r / R_{\odot}$. The radius of the Sun is

$$
R_{\odot}=6.96 \times 10^{8} \mathrm{~m}
$$

\footnotetext{
${ }^{2} C P$ violation is an interplay between the neutrino mass matrix and the charged lepton mass matrix. In particular, a real neutrino mass matrix does not preclude $C P$ violation.
}

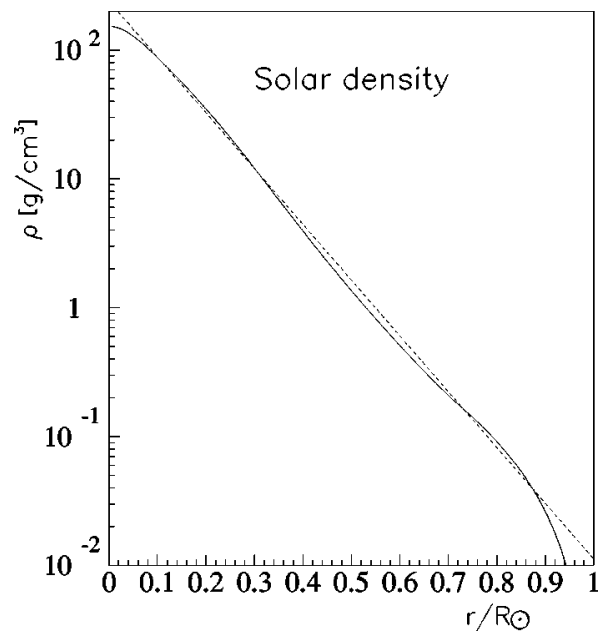

FIG. 1. Density profile of the Sun [10], with exponential approximation (dashed)

From this solid curve in Fig. 1, it is seen that the solar density is approximately exponential, as shown by the dotted line of Fig. 1. This fit is reasonably good, to an accuracy of roughly $15 \%$, for

$$
0.05<r / R_{\odot}<0.9 \text {. }
$$

In other words, $D(r)$ is taken to be given by

$$
D(r)=D(0) e^{-r / r_{0}} .
$$

From the dotted line of Fig. 1, the following values are obtained

$$
D(0)=0.0458 \mathrm{~km}^{-1}, \quad r_{0} \simeq 0.1 \times R_{\odot} .
$$

The problem is therefore to solve the MSW differential equation, for $r \geqslant 0$,

$$
\begin{aligned}
i \frac{d}{d r}\left[\begin{array}{l}
\phi_{1}(r) \\
\phi_{2}(r) \\
\phi_{3}(r)
\end{array}\right]= & \frac{1}{2 p}\left[\begin{array}{ccc}
2 p D(0) e^{-r / r_{0}+M_{11}^{2}} & M_{12}^{2} & M_{13}^{2} \\
M_{21}^{2} & M_{22}^{2} & M_{23}^{2} \\
M_{31}^{2} & M_{32}^{2} & M_{33}^{2}
\end{array}\right] \\
& \times\left[\begin{array}{l}
\phi_{1}(r) \\
\phi_{2}(r) \\
\phi_{3}(r)
\end{array}\right]
\end{aligned}
$$

with the boundary conditions

$$
\left[\begin{array}{l}
\phi_{1}(0) \\
\phi_{2}(0) \\
\phi_{3}(0)
\end{array}\right]=\left[\begin{array}{l}
1 \\
0 \\
0
\end{array}\right] .
$$

\section{SOLUTION OF THE DIFFERENTIAL EQUATION}

The differential equation (2.8) can be solved explicitly in terms of generalized hypergeometric functions. This is to be carried out in the present section. Indeed, this is the underlying reason for the choice of the exponential function (2.6): Let 


$$
u=r / r_{0}+u_{0}
$$

with

$$
u_{0}=-\ln \left[D(0) r_{0}\right] \sim-8.07 \text {. }
$$

Then Eq. (2.8) is

$$
i \frac{d}{d u}\left[\begin{array}{c}
\phi_{1} \\
\phi_{2} \\
\phi_{3}
\end{array}\right]=\left[\begin{array}{ccc}
e^{-u}+\frac{r_{0}}{2 p} M_{11}^{2} & \frac{r_{0}}{2 p} M_{12}^{2} & \frac{r_{0}}{2 p} M_{13}^{2} \\
\frac{r_{0}}{2 p} M_{21}^{2} & \frac{r_{0}}{2 p} M_{22}^{2} & \frac{r_{0}}{2 p} M_{23}^{2} \\
\frac{r_{0}}{2 p} M_{31}^{2} & \frac{r_{0}}{2 p} M_{32}^{2} & \frac{r_{0}}{2 p} M_{33}^{2}
\end{array}\right]\left[\begin{array}{c}
\phi_{1} \\
\phi_{2} \\
\phi_{3}
\end{array}\right] .
$$

Note that $u=0$ corresponds to

$$
r=-r_{0} u_{0} \sim 0.81 R_{\odot}
$$

In view of the form of Eq. (3.3), it is convenient to diagonalize the $2 \times 2$ matrix

$$
\frac{r_{0}}{2 p}\left[\begin{array}{ll}
M_{22}^{2} & M_{23}^{2} \\
M_{32}^{2} & M_{33}^{2}
\end{array}\right]
$$

Let $\theta_{0}$ be the angle of rotation that diagonalizes this $2 \times 2$ matrix, i.e.,

$$
\begin{aligned}
& {\left[\begin{array}{cc}
\cos \theta_{0} & -\sin \theta_{0} \\
\sin \theta_{0} & \cos \theta_{0}
\end{array}\right] \frac{r_{0}}{2 p}\left[\begin{array}{ll}
M_{22}^{2} & M_{23}^{2} \\
M_{32}^{2} & M_{33}^{2}
\end{array}\right]\left[\begin{array}{cc}
\cos \theta_{0} & \sin \theta_{0} \\
-\sin \theta_{0} & \cos \theta_{0}
\end{array}\right]} \\
& \quad=\left[\begin{array}{cc}
\omega_{2} & 0 \\
0 & \omega_{3}
\end{array}\right]
\end{aligned}
$$

with

$$
\omega_{2}<\omega_{3}
$$

The reason for this Eq. (3.6) is that, if $\omega_{2}=\omega_{3}$, then the problem reduces to neutrino oscillations with only two neutrinos. Define also

$$
\omega_{1}=\frac{r_{0}}{2 p} M_{11}^{2}, \quad\left[\begin{array}{l}
\chi_{2} \\
\chi_{3}
\end{array}\right]=\frac{r_{0}}{2 p}\left[\begin{array}{cc}
\cos \theta_{0} & -\sin \theta_{0} \\
\sin \theta_{0} & \cos \theta_{0}
\end{array}\right]\left[\begin{array}{l}
M_{12}^{2} \\
M_{13}^{2}
\end{array}\right]
$$

and

$$
\psi_{1}(u)=\phi_{1}(u), \quad\left[\begin{array}{l}
\psi_{2}(u) \\
\psi_{3}(u)
\end{array}\right]=\left[\begin{array}{cc}
\cos \theta_{0} & -\sin \theta_{0} \\
\sin \theta_{0} & \cos \theta_{0}
\end{array}\right]\left[\begin{array}{l}
\phi_{2}(u) \\
\phi_{3}(u)
\end{array}\right]
$$

then Eq. (3.3) becomes

$$
i \frac{d}{d u}\left[\begin{array}{c}
\psi_{1}(u) \\
\psi_{2}(u) \\
\psi_{3}(u)
\end{array}\right]=\left[\begin{array}{ccc}
\omega_{1}+e^{-u} & \chi_{2} & \chi_{3} \\
\chi_{2} & \omega_{2} & 0 \\
\chi_{3} & 0 & \omega_{3}
\end{array}\right]\left[\begin{array}{l}
\psi_{1}(u) \\
\psi_{2}(u) \\
\psi_{3}(u)
\end{array}\right] .
$$

If $\chi_{2}$ or $\chi_{3}$ is zero, then once again this reduces to neutrino oscillations with two generations. By reversing the signs of $\psi_{2}(u)$ and/or $\psi_{3}(u)$ if necessary, it follows that, without loss of generality

$$
\chi_{j}>0, \quad \text { for } j=2,3 \text {. }
$$

Let $\mu_{1}, \mu_{2}$, and $\mu_{3}$ be the eigenvalues of the $3 \times 3$ matrix

$$
\left[\begin{array}{ccc}
\omega_{1} & \chi_{2} & \chi_{3} \\
\chi_{2} & \omega_{2} & 0 \\
\chi_{3} & 0 & \omega_{3}
\end{array}\right]
$$

then these three $\mu$ 's are the squares of the neutrino masses multiplied by $r_{0} /(2 p)$. Note that

$$
\sum_{j=1}^{3} \omega_{j}=\sum_{j=1}^{3} \mu_{j}
$$

For cases of interest [1], the $\mu_{j}$ will typically be large, $\mu_{j}$ $\gg 1$. This is of course not a question of units, but rather a reflection of the physical situation of having a large number of oscillations within the solar radius.

The interlacing property implies that, by renumbering the $\mu$ 's if necessary,

$$
0 \leqslant \mu_{1}<\omega_{2}<\mu_{2}<\omega_{3}<\mu_{3},
$$

again assuming that there is no reduction to two-neutrino oscillation.

The following properties are easily verified: With fixed $\omega_{2}, \omega_{3}, \chi_{2}$ and $\chi_{3}$,

(i) $\frac{\partial \mu_{j}}{\partial \omega_{1}}>0 \quad$ for $\quad j=1,2,3$

(ii) As $\omega_{1} \rightarrow \infty$,

$$
\mu_{1} \rightarrow \omega_{2}, \quad \mu_{2} \rightarrow \omega_{3}, \quad \text { and } \quad \mu_{3} \rightarrow \infty
$$

(iii) As $\omega_{1} \rightarrow-\infty$, which is physically not possible,

$$
\mu_{1} \rightarrow-\infty, \quad \mu_{2} \rightarrow \omega_{2}, \quad \text { and } \mu_{3} \rightarrow \omega_{3} .
$$

A third-order ordinary differential equation can be obtained from Eq. (3.9) as follows. Define $\psi_{11}, \psi_{22}$, and $\psi_{33}$ such that 


$$
\begin{aligned}
& \psi_{1}=\left(i \frac{d}{d u}-\omega_{2}\right)\left(i \frac{d}{d u}-\omega_{3}\right) \psi_{11}, \\
& \psi_{2}=\chi_{2}\left(i \frac{d}{d u}-\omega_{3}\right) \psi_{22}, \\
& \psi_{3}=\chi_{3}\left(i \frac{d}{d u}-\omega_{2}\right) \psi_{33} .
\end{aligned}
$$

These $\psi_{11}, \psi_{22}$, and $\psi_{33}$ are of course not unique. It then follows from Eqs. (3.9) and (3.10) that

$$
\begin{aligned}
& \left(i \frac{d}{d u}-\omega_{2}\right)\left(i \frac{d}{d u}-\omega_{3}\right)\left(\psi_{11}-\psi_{22}\right)=0, \\
& \left(i \frac{d}{d u}-\omega_{2}\right)\left(i \frac{d}{d u}-\omega_{3}\right)\left(\psi_{11}-\psi_{33}\right)=0 .
\end{aligned}
$$

Therefore,

$$
\begin{aligned}
& \psi_{22}=\psi_{11}+K_{22} e^{-i \omega_{2} u}+K_{23} e^{-i \omega_{3} u}, \\
& \psi_{33}=\psi_{11}+K_{32} e^{-i \omega_{2} u}+K_{33} e^{-i \omega_{3} u} .
\end{aligned}
$$

In particular,

$$
\psi_{22}-\left(K_{23}-K_{33}\right) e^{-i \omega_{3} u}=\psi_{33}-\left(K_{32}-K_{22}\right) e^{-i \omega_{2} u} .
$$

Define this function of Eq. (3.16) to be $\psi$, then it follows that

$$
\begin{aligned}
& \psi_{11}=\psi-K_{22} e^{-i \omega_{2} u}-K_{33} e^{-i \omega_{3} u}, \\
& \psi_{22}=\psi+\left(K_{23}-K_{33}\right) e^{-i \omega_{3} u}, \\
& \psi_{33}=\psi+\left(K_{32}-K_{22}\right) e^{-i \omega_{2} u} .
\end{aligned}
$$

Substitution into Eq. (3.13) then expresses $\psi_{1}, \psi_{2}$, and $\psi_{3}$ in terms of the single function $\psi$ :

$$
\begin{aligned}
& \psi_{1}=\left(i \frac{d}{d u}-\omega_{2}\right)\left(i \frac{d}{d u}-\omega_{3}\right) \psi, \\
& \psi_{2}=\chi_{2}\left(i \frac{d}{d u}-\omega_{3}\right) \psi \\
& \psi_{3}=\chi_{3}\left(i \frac{d}{d u}-\omega_{2}\right) \psi
\end{aligned}
$$

Furthermore, this $\psi$ is unique because of Eq. (3.6).

To obtain the third-order ordinary differential equation for $\psi$, it only remains to substitute Eq. (3.18) into the first equation of Eq. (3.9):

$$
\begin{gathered}
\left(i \frac{d}{d u}-\omega_{1}-e^{-u}\right)\left(i \frac{d}{d u}-\omega_{2}\right)\left(i \frac{d}{d u}-\omega_{3}\right) \psi \\
=\left[\chi_{2}^{2}\left(i \frac{d}{d u}-\omega_{3}\right)+\chi_{3}^{2}\left(i \frac{d}{d u}-\omega_{2}\right)\right] \psi .
\end{gathered}
$$

From the definition of $\mu_{1}, \mu_{2}$, and $\mu_{3}$, this can be rewritten as

$$
\begin{gathered}
\left(i \frac{d}{d u}-\mu_{1}\right)\left(i \frac{d}{d u}-\mu_{2}\right)\left(i \frac{d}{d u}-\mu_{3}\right) \psi \\
=e^{-u}\left(i \frac{d}{d u}-\omega_{2}\right)\left(i \frac{d}{d u}-\omega_{3}\right) \psi
\end{gathered}
$$

To put this equation into the form of the differential equation for the generalized hypergeometric function, let

$$
z=i e^{-u}
$$

then the differential equation (3.20) for $\psi$ is

$$
\begin{gathered}
{\left[\left(z \frac{d}{d z}-i \mu_{1}\right)\left(z \frac{d}{d z}-i \mu_{2}\right)\left(z \frac{d}{d z}-i \mu_{3}\right)\right.} \\
\left.-z\left(z \frac{d}{d z}-i \omega_{2}\right)\left(z \frac{d}{d z}-i \omega_{3}\right)\right] \psi=0 .
\end{gathered}
$$

This is the differential equation for the generalized hypergeometric function ${ }_{2} F_{2}$-see, for example, p. 184 of [11]. Three linearly independent solutions of this third-order differential equation (3.22) are

$\psi^{(1)}=K_{1} e^{-i \mu_{1} u}{ }_{2} F_{2}\left[\begin{array}{cc}-i\left(\omega_{2}-\mu_{1}\right), & -i\left(\omega_{3}-\mu_{1}\right) \\ 1-i\left(\mu_{2}-\mu_{1}\right), & 1-i\left(\mu_{3}-\mu_{1}\right)\end{array} \mid i e^{-u}\right]$,

$\psi^{(2)}=K_{2} e^{-i \mu_{2} u}{ }_{2} F_{2}\left[\begin{array}{cc}-i\left(\omega_{2}-\mu_{2}\right), & -i\left(\omega_{3}-\mu_{2}\right) \\ 1-i\left(\mu_{1}-\mu_{2}\right), & 1-i\left(\mu_{3}-\mu_{2}\right)\end{array} \mid i e^{-u}\right]$,

$\psi^{(3)}=K_{3} e^{-i \mu_{3} u}{ }_{2} F_{2}\left[\begin{array}{cc}-i\left(\omega_{2}-\mu_{3}\right), & -i\left(\omega_{3}-\mu_{3}\right) \\ 1-i\left(\mu_{1}-\mu_{3}\right), & 1-i\left(\mu_{2}-\mu_{3}\right)\end{array} \mid i e^{-u}\right]$,

where $K_{1}, K_{2}$, and $K_{3}$ are arbitrary non-zero constants. For the convenience of numerical calculations, we make the following choice:

$$
\begin{aligned}
& K_{1}=\left[\left(\omega_{2}-\mu_{1}\right)\left(\omega_{3}-\mu_{1}\right)\left(\mu_{2}-\mu_{1}\right)\left(\mu_{3}-\mu_{1}\right)\right]^{-1 / 2}, \\
& K_{2}=\left[\left(\omega_{2}-\mu_{2}\right)\left(\omega_{3}-\mu_{2}\right)\left(\mu_{1}-\mu_{2}\right)\left(\mu_{3}-\mu_{2}\right)\right]^{-1 / 2}, \\
& K_{3}=\left[\left(\omega_{2}-\mu_{3}\right)\left(\omega_{3}-\mu_{3}\right)\left(\mu_{1}-\mu_{3}\right)\left(\mu_{2}-\mu_{3}\right)\right]^{-1 / 2} .
\end{aligned}
$$

The function ${ }_{2} F_{2}$ can be defined by the following series expansion $^{3}[11]$

\footnotetext{
${ }^{3}$ From this form, it is also rather obvious how the confluent hypergeometric function ${ }_{1} F_{1}$ emerges as the solution when one of the $\alpha$ 's equals one of the $\rho$ 's, i.e., for the case of two generations.
} 


$$
{ }_{2} F_{2}\left[\begin{array}{cc}
\alpha_{1}, & \alpha_{2} \\
\rho_{1}, & \rho_{2}
\end{array} \mid z\right]=\sum_{k=0}^{\infty} \frac{\left(\alpha_{1}\right)_{k}\left(\alpha_{2}\right)_{k}}{\left(\rho_{1}\right)_{k}\left(\rho_{2}\right)_{k}} \frac{z^{k}}{k !},
$$

where the Pochhammer symbol is defined as

$$
(\alpha)_{k}=\alpha(\alpha+1) \ldots(\alpha+k-1) .
$$

Since the Taylor series of ${ }_{2} F_{2}$ about $z=0$, Eq. (3.25), is always convergent, the differentiations specified in Eqs. (3.18) can be carried out term by term on these Taylor series.
Thus these derivatives of $\psi^{(j)}$ can be easily expressed in terms of ${ }_{2} F_{2}$ with parameters slightly modified. The result is

$$
\begin{aligned}
& \psi_{1}=C_{1} \psi_{1}^{(1)}+C_{2} \psi_{1}^{(2)}+C_{3} \psi_{1}^{(3)}, \\
& \psi_{2}=C_{1} \psi_{2}^{(1)}+C_{2} \psi_{2}^{(2)}+C_{3} \psi_{2}^{(3)}, \\
& \psi_{3}=C_{1} \psi_{3}^{(1)}+C_{2} \psi_{3}^{(2)}+C_{3} \psi_{3}^{(3)},
\end{aligned}
$$

where

$$
\begin{aligned}
& \psi_{1}^{(1)}=K_{1}\left(\mu_{1}-\omega_{2}\right)\left(\mu_{1}-\omega_{3}\right) e^{-i \mu_{1}{ }_{2} F_{2}}\left[\begin{array}{ll}
1-i\left(\omega_{2}-\mu_{1}\right), & 1-i\left(\omega_{3}-\mu_{1}\right) \\
1-i\left(\mu_{2}-\mu_{1}\right), & 1-i\left(\mu_{3}-\mu_{1}\right)
\end{array} \mid i e^{-u}\right], \\
& \psi_{1}^{(2)}=K_{2}\left(\mu_{2}-\omega_{2}\right)\left(\mu_{2}-\omega_{3}\right) e^{-i \mu_{2} u}{ }_{2} F_{2}\left[\begin{array}{ll}
1-i\left(\omega_{2}-\mu_{2}\right), & 1-i\left(\omega_{3}-\mu_{2}\right) \\
1-i\left(\mu_{1}-\mu_{2}\right), & 1-i\left(\mu_{3}-\mu_{2}\right)
\end{array} \mid i e^{-u}\right] \text {, } \\
& \psi_{1}^{(3)}=K_{3}\left(\mu_{3}-\omega_{2}\right)\left(\mu_{3}-\omega_{3}\right) e^{-i \mu_{3} u}{ }_{2} F_{2}\left[\begin{array}{ll}
1-i\left(\omega_{2}-\mu_{3}\right), & 1-i\left(\omega_{3}-\mu_{3}\right) \\
1-i\left(\mu_{1}-\mu_{3}\right), & 1-i\left(\mu_{2}-\mu_{3}\right)
\end{array} \mid i e^{-u}\right] \text {, } \\
& \psi_{2}^{(1)}=K_{1} \chi_{2}\left(\mu_{1}-\omega_{3}\right) e^{-i \mu_{1} u_{2} F_{2}}\left[\begin{array}{cc}
-i\left(\omega_{2}-\mu_{1}\right), & 1-i\left(\omega_{3}-\mu_{1}\right) \\
1-i\left(\mu_{2}-\mu_{1}\right), & 1-i\left(\mu_{3}-\mu_{1}\right)
\end{array} \mid i e^{-u}\right], \\
& \psi_{2}^{(2)}=K_{2} \chi_{2}\left(\mu_{2}-\omega_{3}\right) e^{-i \mu_{2} u}{ }_{2} F_{2}\left[\begin{array}{cc}
-i\left(\omega_{2}-\mu_{2}\right), & 1-i\left(\omega_{3}-\mu_{2}\right) \\
1-i\left(\mu_{1}-\mu_{2}\right), & 1-i\left(\mu_{3}-\mu_{2}\right)
\end{array} \mid i e^{-u}\right] \text {, } \\
& \psi_{2}^{(3)}=K_{3} \chi_{2}\left(\mu_{3}-\omega_{3}\right) e^{-i \mu_{3} u}{ }_{2} F_{2}\left[\begin{array}{cc}
-i\left(\omega_{2}-\mu_{3}\right), & 1-i\left(\omega_{3}-\mu_{3}\right) \\
1-i\left(\mu_{1}-\mu_{3}\right), & 1-i\left(\mu_{2}-\mu_{3}\right)
\end{array} \mid i e^{-u}\right] \text {, } \\
& \psi_{3}^{(1)}=K_{1} \chi_{3}\left(\mu_{1}-\omega_{2}\right) e^{-i \mu_{1} u_{2} F_{2}}\left[\begin{array}{cc}
1-i\left(\omega_{2}-\mu_{1}\right), & -i\left(\omega_{3}-\mu_{1}\right) \\
1-i\left(\mu_{2}-\mu_{1}\right), & 1-i\left(\mu_{3}-\mu_{1}\right)
\end{array} \mid i e^{-u}\right], \\
& \psi_{3}^{(2)}=K_{2} \chi_{3}\left(\mu_{2}-\omega_{2}\right) e^{-i \mu_{2} u}{ }_{2} F_{2}\left[\begin{array}{cc}
1-i\left(\omega_{2}-\mu_{2}\right), & -i\left(\omega_{3}-\mu_{2}\right) \\
1-i\left(\mu_{1}-\mu_{2}\right), & 1-i\left(\mu_{3}-\mu_{2}\right)
\end{array} \mid i e^{-u}\right], \\
& \psi_{3}^{(3)}=K_{3} \chi_{3}\left(\mu_{3}-\omega_{2}\right) e^{-i \mu_{3} u}{ }_{2} F_{2}\left[\begin{array}{cc}
1-i\left(\omega_{2}-\mu_{3}\right), & -i\left(\omega_{3}-\mu_{3}\right) \\
1-i\left(\mu_{1}-\mu_{3}\right), & 1-i\left(\mu_{2}-\mu_{3}\right)
\end{array} \mid i e^{-u}\right] .
\end{aligned}
$$

In Eqs. (3.27), the constants $C_{1}, C_{2}$ and $C_{3}$ are determined by the boundary conditions Eqs. (2.9). For the exponential matter distribution (2.6), this gives completely the solution of Eq. (2.4), which describes the MSW effect in the sun.

We recall that by Eq. (3.2), $e^{-u}=r_{0} D(0) e^{-r / r_{0}}$. Thus, the prefactors $e^{-i \mu_{j} u}$ are proportional to the exponentials describing propagation in vacuum, $e^{-i \mu_{j} r / r_{0}}$. In fact, as $r \rightarrow \infty$, all these functions approach a simple limit:

$$
\lim _{u \rightarrow \infty} F_{2}\left[\begin{array}{cc}
\alpha_{1}, & \alpha_{2} \\
\rho_{1}, & \rho_{2}
\end{array} \mid i e^{-u}\right]=1 .
$$

While this solution (3.27) is specifically for three generations of neutrinos, generalization, if desired, to $N$ generations is completely straightforward, leading to the generalized hypergeometric function ${ }_{N-1} F_{N-1}$. Even for three generations, ${ }_{2} F_{2}$ is a complicated function. From Eq. (3.2), corresponding to the center of the Sun, $e^{-u}$ is about 3000; for arguments of this order of magnitude, it is not practical to calculate the various ${ }_{2} F_{2}$ of Eqs. (3.28)-(3.30) using the Taylor series expansion.

Sections IV and V are devoted to the issue of how these ${ }_{2} F_{2}$ can be calculated. Of the nine ${ }_{2} F_{2}$ that appear in Eqs. (3.28)-(3.30), three of them are evaluated approximately in Sec. IV. In Sec. V, the remaining six ${ }_{2} F_{2}$ are then expressed in terms of another set of generalized hypergeometric functions ${ }_{3} F_{1}$. These ${ }_{3} F_{1}$ have convenient integral representations, useful for numerical evaluation. The approach de- 
scribed in the rest of this paper is the best we have found, but there may well be other methods that are superior.

\section{IV. ${ }_{2} F_{2}$ THAT APPEAR IN $\psi_{j}^{(3)}$}

In this section, we study the three ${ }_{2} F_{2}$ that appear in the expressions of $\psi_{j}^{(1)}, \psi_{j}^{(2)}$, and $\psi_{j}^{(3)}$ as given by Eqs. (3.28)(3.30). Define

$$
\begin{array}{ll}
\rho_{1}=\mu_{3}-\mu_{1}, & \rho_{2}=\mu_{3}-\omega_{2}, \\
\rho_{3}=\mu_{3}-\mu_{2}, & \rho_{4}=\mu_{3}-\omega_{3} ;
\end{array}
$$

then by Eq. (3.12)

$$
\rho_{1}>\rho_{2}>\rho_{3}>\rho_{4}>0,
$$

and the three ${ }_{2} F_{2}$ of interest are

$$
\begin{aligned}
& { }_{2} F_{2}(1)={ }_{2} F_{2}\left[\begin{array}{ll}
1+i \rho_{2}, & 1+i \rho_{4} \\
1+i \rho_{1}, & 1+i \rho_{3}
\end{array} \mid i e^{-u}\right], \\
& { }_{2} F_{2}(2)={ }_{2} F_{2}\left[\begin{array}{cc}
i \rho_{2}, & 1+i \rho_{4} \\
1+i \rho_{1}, & 1+i \rho_{3}
\end{array} \mid i e^{-u}\right] \text {, } \\
& { }_{2} F_{2}(3)={ }_{2} F_{2}\left[\begin{array}{cc}
1+i \rho_{2}, & i \rho_{4} \\
1+i \rho_{1}, & 1+i \rho_{3}
\end{array} \mid i e^{-u}\right] \text {. }
\end{aligned}
$$

Let ${ }_{2} F_{2}(1)$ be considered in some detail; the treatments of ${ }_{2} F_{2}(2)$ and ${ }_{2} F_{2}(3)$ are similar.

From the definition of ${ }_{2} F_{2}$ by its Taylor series, which is always convergent, it is straightforward to verify, by closing the contour of integration in the right half-plane, that

$$
\begin{aligned}
{ }_{2} F_{2}(1)= & \frac{1}{2 \pi i} \frac{\Gamma\left(1+i \rho_{1}\right) \Gamma\left(1+i \rho_{3}\right)}{\Gamma\left(1+i \rho_{2}\right) \Gamma\left(1+i \rho_{4}\right)} \\
& \times \int_{\gamma-i \infty}^{\gamma+i \infty} \frac{\Gamma(-s) \Gamma\left(1+i \rho_{2}+s\right) \Gamma\left(1+i \rho_{4}+s\right)}{\Gamma\left(1+i \rho_{1}+s\right) \Gamma\left(1+i \rho_{3}+s\right)} \\
& \times\left(e^{-i \pi / 2} e^{-u}\right)^{s} d s
\end{aligned}
$$

where

$$
-1<\gamma<0
$$

This integral is to be evaluated approximately for $u$ large and negative. Except for an indentation near $s=0$, the contour of integration can be taken along the imaginary axis. It is therefore convenient to let

$$
s=i \tau \text {. }
$$

The Stirling formula

$$
\Gamma(z) \sim(2 \pi)^{1 / 2} e^{-z} e^{(z-1 / 2) \ln z}
$$

takes the form

$$
\Gamma(i x) \sim\left(\frac{2 \pi}{i x}\right)^{1 / 2} e^{-\pi|x| / 2} e^{i x(\ln |x|-1)}
$$

when $z$ is purely imaginary. Using Eq. (4.8), the integrand in Eq. (4.4) is

$$
\begin{aligned}
& \frac{\Gamma(-s) \Gamma\left(1+i \rho_{2}+s\right) \Gamma\left(1+i \rho_{4}+s\right)}{\Gamma\left(1+i \rho_{1}+s\right) \Gamma\left(1+i \rho_{3}+s\right)}\left(e^{-i \pi / 2} e^{-u}\right)^{s} \\
& =\frac{\Gamma(-i \tau) \Gamma\left(1+i\left(\tau+\rho_{2}\right)\right) \Gamma\left(1+i\left(\tau+\rho_{4}\right)\right)}{\Gamma\left(1+i\left(\tau+\rho_{1}\right)\right) \Gamma\left(1+i\left(\tau+\rho_{3}\right)\right)} e^{\pi \tau / 2} e^{i|u| \tau} \\
& \sim\left(\frac{2 \pi}{-i \tau}\right)^{1 / 2} \frac{\left[i\left(\tau+\rho_{2}\right)\right]^{1 / 2}\left[i\left(\tau+\rho_{4}\right)\right]^{1 / 2}}{\left[i\left(\tau+\rho_{1}\right)\right]^{1 / 2}\left[i\left(\tau+\rho_{3}\right)\right]^{1 / 2}} e^{\pi \theta_{1}(\tau) / 2} e^{i \theta_{2}(\tau)},
\end{aligned}
$$

with

$$
\theta_{1}(\tau)=\left|\tau+\rho_{1}\right|-\left|\tau+\rho_{2}\right|+\left|\tau+\rho_{3}\right|-\left|\tau+\rho_{4}\right|-|\tau|+\tau,
$$

and

$$
\begin{aligned}
\theta_{2}(\tau)= & -\tau(\ln |\tau|-1)-\left(\tau+\rho_{1}\right)\left(\ln \left|\tau+\rho_{1}\right|-1\right)+\left(\tau+\rho_{2}\right) \\
& \times\left(\ln \left|\tau+\rho_{2}\right|-1\right)-\left(\tau+\rho_{3}\right)\left(\ln \left|\tau+\rho_{3}\right|-1\right) \\
& +\left(\tau+\rho_{4}\right)\left(\ln \left|\tau+\rho_{4}\right|-1\right)+|u| \tau
\end{aligned}
$$

The condition

$$
\frac{\partial \theta_{2}(\tau)}{\partial \tau}=0
$$

for stationary phase gives

$$
\frac{|\tau|\left|\tau+\rho_{1}\right|\left|\tau+\rho_{3}\right|}{\left|\tau+\rho_{2}\right|\left|\tau+\rho_{4}\right|}=e^{|u|} .
$$

Equation (4.13) together with the fact that $\theta_{1}(\tau)$ is a nondecreasing function of $\tau$ implies that the relevant point of stationary phase occurs when $\tau$ is positive. Let $\tau_{0}$ be the unique positive root of the cubic equation

$$
\frac{\tau_{0}\left(\tau_{0}+\rho_{1}\right)\left(\tau_{0}+\rho_{3}\right)}{\left(\tau_{0}+\rho_{2}\right)\left(\tau_{0}+\rho_{4}\right)}=e^{|u|},
$$

then $\tau_{0}$ is the point of stationary phase that gives the leading contribution to ${ }_{2} F_{2}(1)$. The result is, with all gamma functions replaced by the corresponding Stirling formula (4.7),

$$
\begin{aligned}
{ }_{2} F_{2}(1) \sim & \left(\frac{\rho_{1} \rho_{3}}{\rho_{2} \rho_{4}}\right)^{1 / 2}\left[\frac{\left(\tau_{0}+\rho_{2}\right)\left(\tau_{0}+\rho_{4}\right)}{\left(\tau_{0}+\rho_{1}\right)\left(\tau_{0}+\rho_{3}\right)}\right]^{1 / 2} \\
& \times e^{i\left[\rho_{1}\left(\ln \rho_{1}-1\right)-\rho_{2}\left(\ln \rho_{2}-1\right)+\rho_{3}\left(\ln \rho_{3}-1\right)-\rho_{4}\left(\ln \rho_{4}-1\right)\right]} \\
& \times\left[1+\frac{\tau_{0}}{\tau_{0}+\rho_{1}}-\frac{\tau_{0}}{\tau_{0}+\rho_{2}}+\frac{\tau_{0}}{\tau_{0}+\rho_{3}}-\frac{\tau_{0}}{\tau_{0}+\rho_{4}}\right]^{-1 / 2} \\
& \times e^{i \theta_{2}\left(\tau_{0}\right)},
\end{aligned}
$$

for $u$ negative and large. Similarly,

$$
{ }_{2} F_{2}(2) \sim \frac{\rho_{2}}{\tau_{0}+\rho_{2}}{ }_{2} F_{2}(1),
$$




$$
{ }_{2} F_{2}(3) \sim \frac{\rho_{4}}{\tau_{0}+\rho_{4}}{ }_{2} F_{2}(1) .
$$

These are the desired results.

\section{V. ${ }_{2} F_{2}$ THAT APPEAR IN $\psi_{j}^{(1)}$ AND $\psi_{j}^{(2)}$}

The next step is to study the ${ }_{2} F_{2}$ that appear in the expressions (3.28)-(3.30) for $\psi_{1}^{(i)}, \psi_{2}^{(i)}$, and $\psi_{3}^{(i)}$ with $i=1,2$. It should be emphasized that, while the treatment in the preceding Sec. IV involves approximations, what is to be carried out in this section is exact. Since exact manipulations are usually more straightforward, the description will be relatively more brief in this section.

Consider the third-order ordinary differential equation

$$
\begin{array}{r}
{\left[\left(z \frac{d}{d z}+\beta_{1}\right)\left(z \frac{d}{d z}+\beta_{2}\right)\left(z \frac{d}{d z}+\beta_{3}\right)\right.} \\
\left.-z\left(z \frac{d}{d z}+\alpha_{1}\right)\left(z \frac{d}{d z}+\alpha_{2}\right)\right] f=0 .
\end{array}
$$

A comparison with Eq. (3.22) shows that

$$
\beta_{1}=-i \mu_{1}, \quad \beta_{2}=-i \mu_{2}, \quad \beta_{3}=-i \mu_{3} .
$$

The values of $\alpha_{1}$ and $\alpha_{2}$ are different for the various $\psi$ 's:

$$
\begin{array}{llll}
\text { for } \psi_{1}^{(j)}: & \alpha_{1}=1-i \omega_{2} \quad \text { and } & \alpha_{2}=1-i \omega_{3}, \\
\text { for } \psi_{2}^{(j)}: & \alpha_{1}=-i \omega_{2} \text { and } & \alpha_{2}=1-i \omega_{3}, \\
\text { for } \psi_{3}^{(j)}: & \alpha_{1}=1-i \omega_{2} \text { and } & \alpha_{2}=-i \omega_{3}, \\
\text { for } \psi^{(j)}: & \alpha_{1}=-i \omega_{2} \text { and } & \alpha_{2}=-i \omega_{3} .
\end{array}
$$

The three linearly independent solutions of Eq. (5.1) are

$$
\begin{aligned}
& f^{(1)}(z)=z^{-\beta_{1} F_{2}}\left[\begin{array}{cc}
\alpha_{1}-\beta_{1}, & \alpha_{2}-\beta_{1} \\
1+\beta_{2}-\beta_{1}, & 1+\beta_{3}-\beta_{1}
\end{array} \mid z\right], \\
& f^{(2)}(z)=z^{-\beta_{2} F_{2}}\left[\begin{array}{cc}
\alpha_{1}-\beta_{2}, & \alpha_{2}-\beta_{2} \\
1+\beta_{1}-\beta_{2}, & 1+\beta_{3}-\beta_{2}
\end{array} \mid z\right], \\
& f^{(3)}(z)=z^{-\beta_{3} F_{2}}\left[\begin{array}{cc}
\alpha_{1}-\beta_{3}, & \alpha_{2}-\beta_{3} \\
1+\beta_{1}-\beta_{3}, & 1+\beta_{2}-\beta_{3}
\end{array} \mid z\right],
\end{aligned}
$$

where $f^{(3)}(z)$ is the function treated in the preceding Sec. IV.

On the other hand, if in Eq. (5.1) the independent variable is changed to

$$
\hat{z}=z^{-1}
$$

then the differential equation is

$$
\left[\left(\hat{z} \frac{d}{d \hat{z}}-\alpha_{1}\right)\left(\hat{z} \frac{d}{d \hat{z}}-\alpha_{2}\right)\right.
$$

$$
\left.+\hat{z}\left(\hat{z} \frac{d}{d \hat{z}}-\beta_{1}\right)\left(\hat{z} \frac{d}{d \hat{z}}-\beta_{2}\right)\left(\hat{z} \frac{d}{d \hat{z}}-\beta_{3}\right)\right] f=0 .
$$

In this form, two of the three linearly independent solutions of Eq. (5.1) are (in terms of $z$ )

$$
\begin{aligned}
& \hat{f}^{(1)}(z)=z^{-\alpha_{1}}
\end{aligned}
$$

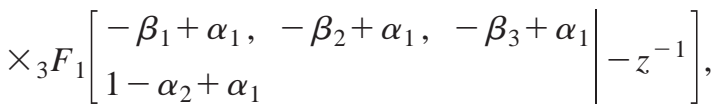

$$
\begin{aligned}
& \hat{f}^{(2)}(z)=z^{-\alpha_{2}}
\end{aligned}
$$

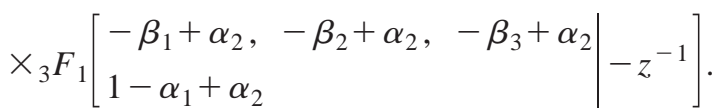

For a discussion of the generalized hypergeometric functions ${ }_{p} F_{q}$ with $p>q+1$, see for example Chap. V of [11].

It should be added parenthetically that, for the present purposes, $z^{-\beta_{1}}$, as an example, can sometimes be very small because by Eqs. (3.21) and (5.2)

$$
z^{-\beta_{1}}=e^{-\pi \mu_{1} / 2} e^{-i \mu_{1} u}
$$

Thus, when $\mu_{1}$ is large, which is often the case, for numerical calculations it is convenient to replace $z^{-\beta_{1}}$ by $e^{-i \mu_{1} u}$, and similarly for the other powers of $z$ multiplying the ${ }_{2} F_{2}$ and ${ }_{3} F_{1}$.

Since Eq. (5.1) is a third-order linear ordinary differential equation, the two solutions as given by Eq. (5.10) must be expressible as linear combinations of the three solutions of Eq. (5.7). The result is

$$
\begin{aligned}
\hat{f}^{(1)}(z)= & \frac{\pi \Gamma\left(1-\alpha_{2}+\alpha_{1}\right)}{\Gamma\left(-\beta_{1}+\alpha_{1}\right) \Gamma\left(-\beta_{2}+\alpha_{1}\right) \Gamma\left(-\beta_{3}+\alpha_{1}\right)} \\
& \times\left[\frac{\Gamma\left(-\beta_{2}+\beta_{1}\right) \Gamma\left(-\beta_{3}+\beta_{1}\right)}{\Gamma\left(1-\alpha_{1}+\beta_{1}\right) \Gamma\left(1-\alpha_{2}+\beta_{1}\right)}\right. \\
& \times \frac{1}{\sin \pi\left(\alpha_{1}-\beta_{1}\right)} f^{(1)}(z) \\
& +\frac{\Gamma\left(-\beta_{1}+\beta_{2}\right) \Gamma\left(-\beta_{3}+\beta_{2}\right)}{\Gamma\left(1-\alpha_{1}+\beta_{2}\right) \Gamma\left(1-\alpha_{2}+\beta_{2}\right)} \\
& \times \frac{1}{\sin \pi\left(\alpha_{1}-\beta_{2}\right)} f^{(2)}(z) \\
& +\frac{\Gamma\left(-\beta_{1}+\beta_{3}\right) \Gamma\left(-\beta_{2}+\beta_{3}\right)}{\Gamma\left(1-\alpha_{1}+\beta_{3}\right) \Gamma\left(1-\alpha_{2}+\beta_{3}\right)} \\
& \left.\times \frac{1}{\sin \pi\left(\alpha_{1}-\beta_{3}\right)} f^{(3)}(z)\right]
\end{aligned}
$$




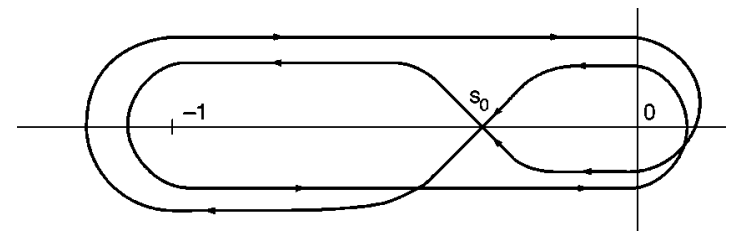

FIG. 2. Pochhammer contour used for the evaluation of the hypergeometric function ${ }_{2} F_{1}$. The point of stationary phase, denoted $s_{0}$, occurs along the path from NE to SW.

$$
\hat{f}^{(2)}(z)=\left.\hat{f}^{(1)}(z)\right|_{\alpha_{1} \leftrightarrow \alpha_{2}} .
$$

Equations (5.12) and (5.13) can be considered to be the formulas that express $f^{(1)}(z)$ and $f^{(2)}(z)$ in terms of $\hat{f}^{(1)}(z)$, $\hat{f}^{(2)}(z)$ and $f^{(3)}(z)$. Since $f^{(3)}(z)$ is given by Eqs. (4.15)(4.17), it only remains to obtain $\hat{f}^{(1)}(z)$ and $\hat{f}^{(2)}(z)$, which are given by ${ }_{3} F_{1}$ rather than ${ }_{2} F_{2}$.

The advantage of ${ }_{3} F_{1}$ over ${ }_{2} F_{2}$ is due to the integral representation

$$
\begin{aligned}
& { }_{3} F_{1}\left[\begin{array}{ccc}
a_{1}, & a_{2}, & a_{3} \\
b & & -x^{-1}
\end{array}\right] \\
& =\frac{1}{\Gamma\left(a_{1}\right)} x^{a_{1}} \int_{0}^{\infty} d t e^{-x t} t^{a_{1}-1}{ }_{2} F_{1}\left(a_{2}, a_{3} ; b ;-t\right),
\end{aligned}
$$

where ${ }_{2} F_{1}$ is the usual hypergeometric function. This Eq. (5.14) follows from Eq. (8) on p. 214 and Eq. (1) on p. 215 of [11], and has the great advantage of not containing in the integrand any gamma function that depends on the variable $t$, because such gamma functions require excessive computer time.

For the ${ }_{2} F_{1}$ in the integrand of Eq. (5.14), the representation

$$
\begin{aligned}
{ }_{2} F_{1}(a, b ; c ;-t)= & \frac{-\Gamma(c) e^{-i \pi c}}{4 \Gamma(b) \Gamma(c-b) \sin \pi b \sin \pi(c-b)} \\
& \times \int_{\mathcal{P}} s^{b-1}(1-s)^{c-b-1}(1+t s)^{-a} d s
\end{aligned}
$$

is convenient, where $\mathcal{P}$ is the Pochhammer contour of Fig. 2. In the numerical evaluation through Eqs. (5.14) and (5.15), it is convenient to deform the contours of integration through the point of stationary phase. This point is discussed in more detail in the Appendix.

\section{SUMMARY AND DISCUSSION}

The Mikheyev-Smirnov-Wolfenstein effect in the sun has been studied and the differential equations solved for three types of neutrinos coupled through their mass matrix. Under the assumption, as expressed by Eq. (2.6), that the electron density can be approximated by an exponential function, the
MSW differential equations are solved exactly in Sec. III, especially Eqs. (3.27)-(3.30), in terms of the generalized hypergeometric function ${ }_{2} F_{2}$. The method used there can be immediately generalized to $N$ types of neutrinos coupled in the same way, leading to ${ }_{N-1} F_{N-1}$.

Since ${ }_{2} F_{2}$ cannot be considered to be a familiar function, Secs. IV and V are devoted to the issue of how they can be calculated. Of the nine ${ }_{2} F_{2}$ that appear in Eqs. (3.28)-(3.30), three are evaluated approximately in Sec. IV. In Sec. V, the remaining six ${ }_{2} F_{2}$ are then expressed in terms of another set of generalized hypergeometric functions, ${ }_{3} F_{1}$. These ${ }_{3} F_{1}$ have convenient integral representations given by Eqs. (5.14) and (5.15), useful for numerical evaluation. Equations (5.14) and (5.15) can also be used to get asymptotic expressions for ${ }_{3} F_{1}$ and hence ${ }_{2} F_{2}$, but this development is not discussed here because it is not needed for the study of neutrino oscillations.

Combining assumption 2 of Sec. I with the result of this paper, it has been found [1] that the mass of the lightest neutrino is $1.3 \times 10^{-3} \mathrm{eV}$, that of the next heavier neutrino is ten times that value, but that of the heaviest neutrino is not well determined. Favored values are in the range 0.03-0.09 $\mathrm{eV}$.

Finally, it should be mentioned that, while the calculation of Sec. IV can be generalized immediately to $N$ types of neutrinos, there does not seem to be a similar straightforward generalization for Sec. V. More precisely, while ${ }_{N-1} F_{N-1}$ can be related to ${ }_{N} F_{N-2}$, the generalization of Eq. (5.14) involves ${ }_{N-1} F_{N-2}$, which does not have an integral representation similar to Eq. (5.15) involving a single integral. We believe that, for ${ }_{N} F_{N-2}$, it is necessary to use an $(N-1)$-fold integral to avoid having gamma functions that depend on the variables of integration.

\section{ACKNOWLEDGMENTS}

This paper owes its existence to the late Professor Harry Lehmann. He introduced us to the issues of mass matrices and participated in the early stages of this work. Also, we would like to thank Professors Evgeny Akhmedov, Alvaro de Rujula, Alexei Smirnov and Gabriele Veneziano, and especially Conrad Newton, for useful discussions. T.T.W. wishes to thank the Theory Division of CERN for its kind hospitality. This work was supported in part by the Research Council of Norway, and in part by the United States Department of Energy under Grant No. DE-FG02-84ER40158.

\section{APPENDIX}

In this appendix, Eqs. (5.14) and (5.15) are studied further. For definiteness, let $\alpha_{1}$ and $\alpha_{2}$ be defined by Eq. (5.3), and consider the ${ }_{3} F_{1}$ that appears in the first equation in Eq. (5.10). Use the inequality (3.12) to define the four positive quantities

$$
\begin{aligned}
& \xi=\mu_{2}-\omega_{2}, \\
& \eta=\omega_{2}-\mu_{1}, \\
& \zeta=\mu_{3}-\omega_{2},
\end{aligned}
$$




$$
\zeta^{\prime}=\omega_{3}-\omega_{2}<\zeta
$$

then Eq. (5.14) gives, for this ${ }_{3} F_{1}$ under consideration,

$$
\begin{aligned}
& { }_{3} F_{1}\left[\begin{array}{cc|c}
1+i \xi, & 1-i \eta, & 1+i \zeta \\
1+i \zeta^{\prime}, & \frac{i}{y}
\end{array}\right] \\
& =\frac{1}{\Gamma(1+i \xi)}\left(e^{i \pi / 2} y\right)^{1+i \xi} \int_{0}^{\infty} d t e^{-i t y} t^{i \xi} \\
& \times{ }_{2} F_{1}\left(1-i \eta, 1+i \zeta ; 1+i \zeta^{\prime} ;-t\right),
\end{aligned}
$$

where, by Eq. (3.21),

$$
y=e^{-u}
$$

is large for $u$ negative and large. The substitution of Eq. (5.15) into Eq. (A2) then gives

$$
\begin{aligned}
& { }_{3} F_{1}\left[\begin{array}{ccc|c}
1+i \xi, & 1-i \eta, & 1+i \zeta & \frac{i}{y} \\
1+i \zeta^{\prime}, &
\end{array}\right] \\
& =\frac{1}{\Gamma(1+i \xi)}\left(e^{i \pi / 2} y\right)^{1+i \xi} \\
& \times \frac{\Gamma\left(1+i \zeta^{\prime}\right) e^{-i \pi\left(1+i \zeta^{\prime}\right)}}{\Gamma(1-i \eta) \Gamma\left(i\left(\eta+\zeta^{\prime}\right)\right) 4 \sin \pi(1-i \eta) \sin \pi i\left(\eta+\zeta^{\prime}\right)} I,
\end{aligned}
$$

where

$$
\begin{aligned}
I & =\int_{0}^{\infty} d t \int_{\mathcal{P}} d s e^{-i t y} t^{i \xi} s^{-i \eta}(1+s)^{i\left(\zeta-\zeta^{\prime}\right)}(1+s+s t)^{-1-i \zeta} \\
& =\int_{0}^{\infty} d t \int_{\mathcal{P}} d s(1+s+s t)^{-1} e^{-i \phi(t, s)},
\end{aligned}
$$

with

$$
\begin{aligned}
\phi(t, s)= & t y-\xi \ln t+\eta \ln s-\left(\zeta-\zeta^{\prime}\right) \ln (1+s) \\
& +\zeta \ln (1+s+s t) .
\end{aligned}
$$

This $\phi$ is of course not related to the $\phi_{j}$ of Eq. (2.4).

For the double integral in Eq. (A5), the point or points of stationary phase are given by

$$
\frac{\partial \phi(t, s)}{\partial t}=\frac{\partial \phi(t, s)}{\partial s}=0
$$

or

$$
y-\frac{\xi}{t}+\frac{s \zeta}{1+s+s t}=0,
$$

and

$$
\frac{\eta}{s}-\frac{\zeta-\zeta^{\prime}}{1+s}+\frac{(1+t) \zeta}{1+s+s t}=0
$$

Elimination of $s$ between Eqs. (A8) and (A9) gives the cubic equation for $t$ :

$$
l(t)=0,
$$

with

$$
\begin{aligned}
l(t)= & y^{2} t^{3}+y(y+\zeta-2 \xi-\eta) t^{2} \\
& +\left[\left(\zeta^{\prime}-2 \xi\right) y-(\zeta-\xi)(\xi+\eta)\right] t-\xi\left(\zeta^{\prime}-\xi\right) .
\end{aligned}
$$

When $\zeta^{\prime} \rightarrow \zeta$, the three roots of Eq. (A10) are explicitly

$$
t=t_{+}=(2 y)^{-1}\left\{-(y-\xi-\eta)+\left[(y-\xi-\eta)^{2}+4 \xi y\right]^{1 / 2}\right\}>0,
$$

$$
t=t_{-}=(2 y)^{-1}\left\{-(y-\xi-\eta)-\left[(y-\xi-\eta)^{2}+4 \xi y\right]^{1 / 2}\right\}<0,
$$

$t=-(\zeta-\xi) / y<0$.

Since, by Eq. (A1)

$$
l\left(t_{-}\right)=-\frac{\eta t_{-}}{1+t_{-}}\left(\zeta-\zeta^{\prime}\right)>0
$$

we have

$$
l(t) \begin{cases}<0 & \text { for } t \text { sufficiently large and negative, } \\ >0 & \text { for } t=t_{-}<0 \\ <0 & \text { for } t=0 \\ >0 & \text { for } t \text { sufficiently large and positive. }\end{cases}
$$

It therefore follows that the cubic equation (A10) has one and only one positive root. Since the $t$ integral in Eq. (A5) is along the positive real axis, this implies that there is exactly one relevant point of stationary phase, say $\left(t_{0}, s_{0}\right)$, with $t_{0}$ given by this unique positive root of Eq. (A10). Furthermore, since

$$
l\left(t_{+}\right)<0,
$$

it follows from Eq. (A16) that

$$
t_{0}>t_{+} .
$$

From Eq. (A8), the corresponding $s_{0}$ is given by

$$
s_{0}=-\left[1+t_{0}+\frac{\zeta t_{0}}{y t_{0}-\xi}\right]^{-1},
$$

and hence

$$
-\left(1+t_{0}\right)^{-1}<s_{0}<0 .
$$

For the numerical evaluation of this ${ }_{3} F_{1}$ of Eq. (A4), using Eq. (A5), it is convenient and efficient to choose the Pochhammer contour $\mathcal{P}$ to go through this value of $s_{0}$, and also to deform the contour for the $t$ integration so that at $t_{0}$ it is locally the path of steepest descent.

The other ${ }_{3} F_{1}$ 's can be treated in similar ways. 
[1] P. Osland and T.T. Wu, CERN Report No. TH/99-285 (1999).

[2] H. Lehmann, C. Newton, and T.T. Wu, Phys. Lett. B 384, 249 (1996).

[3] L. Wolfenstein, Phys. Rev. D 17, 2369 (1978); 20, 2634 (1979); S.P. Mikheyev and A.Yu. Smirnov, Yad. Fiz. 42, 1441 (1985) [Sov. J. Nucl. Phys. 42, 913 (1985)]; Nuovo Cimento C 9, 17 (1986).

[4] Super-Kamiokande Collaboration, Y. Fukuda et al., Phys. Rev. Lett. 81, 1562 (1998).

[5] For an exponential density, see, for example, T. Kaneko, Prog. Theor. Phys. 78, 532 (1987); M. Ito, T. Kaneko, and M. Nakagawa, ibid. 79, 13 (1988); S. Toshev, Phys. Lett. B 196, 170 (1987); S.T. Petcov, ibid. 200, 373 (1988); Nucl. Phys. B (Proc. Suppl.) 13, 527 (1990).

[6] For a linear density, see, for example, W.C. Haxton, Phys. Rev. D 35, 2352 (1987); S.T. Petcov, Phys. Lett. B 191, 299 (1987).

[7] See, for example, T.K. Kuo and J. Pantaleone, Phys. Rev. Lett. 57, 1805 (1986); C.W. Kim and W.K. Sze, Phys. Rev. D 35, 1404 (1987); H.W. Zaglauer and K.H. Schwarzer, Z. Phys. C
40, 273 (1988); G.L. Fogli, E. Lisi, and D. Montanino, Phys. Rev. D 49, 3626 (1994); E. Torrente Lujan, ibid. 53, 4030 (1996); T. Sakai, O. Inagaki, and T. Teshima, Int. J. Mod. Phys. A 12, 1953 (1999); T. Ohlsson and H. Snellman, Royal Inst. Tech., Stockholm, report 1999 hep-ph/9910546; G.L. Fogli, E. Lisi, D. Montanino, and A. Palazzo, Phys. Rev. D 62, 013002 (2000).

[8] S.P. Rosen, in Symmetries and Fundamental Interactions in Nuclei, edited by W.C. Haxton and E.M. Henley (World Scientific, Singapore, 1995), p. 251.

[9] The early papers on the nuclear reactions in the Sun are C.F. von Weizsacker, Phys. Z. 38, 176 (1937); 39, 633 (1938); H.A. Bethe and C.L. Critchfield, Phys. Rev. 54, 248 (1938); 54, 862 (1938); H.A. Bethe, ibid. 55, 434 (1939).

[10] J.N. Bahcall, S. Basu, and M.H. Pinsonneault, Phys. Lett. B 433, 1 (1998); see also J.N. Bahcall's homepage, http://www.sns.ias.edu/ jnb

[11] Bateman Manuscript Project, Higher Transcendental Functions, edited by A. Erdélyi (McGraw-Hill, New York, 1953), Vol. I. 